\title{
Application of Equipment Quality Management in Fulfillment Tasks Based on Alliance Blockchain Intelligent Contract Technology
}

\author{
Xiang $\mathrm{Li}^{1,2}$, Qiwu $\mathrm{Wu}^{1, *}$ and Yongyan $\mathrm{Zhu}^{1}$ \\ ${ }^{1}$ Engineering University of CAPF, Xi'an, 710086, China \\ ${ }^{2}$ A certain department of CAPF, Yulin, Shaanxi, 719000, China \\ *Corresponding author. Email: wuqiwu700@163.com
}

\begin{abstract}
Based on the intelligent contract technology of alliance blockchain, the equipment quality management mode matching responsibility, right and accountability is established. It makes seamless link between the equipment management department and the team which executes the tasks, realizing the intelligent and efficient equipment quality management. This paper mainly focuses on the technical composition and principle through the alliance blockchain using intelligent contract. This paper consists of three aspects, including the analysis of equipment management based on intelligent contract technology, the application of equipment quality management based on the alliance chain intelligent contract technology in order to play a positive role in solving the actual equipment management based on distributed management.
\end{abstract}

Keywords: Alliance blockchain, Smart contracts, Equipment quality management.

\section{INTRODUCTION}

The equipment quality management refers to the equipment support carried out at a certain stage in the process of team carrying out major activities, executing urgent or special tasks, and it is a complex systematic project involving many parties. With the strong scientific and technological support, the pace of equipment upgrading has been greatly accelerated, and the contradiction of the difficulty in ensuring safety, equipment handover and the inadequate supervision caused by the traditional quality management mode for task execution is highlighted. So, the effective combination of efficient task-oriented equipment management and the improvement of real-combat training is of great significance.

Blockchain has the characteristics of decentralization, distributed storage, non-tampering, information transparency, whole-network consensus, etc. Alliance blockchain has the characteristics of openness, low public trust, privacy protection and single high trust of private chain. Alliance chain intelligent contract technology (hereinafter referred to as ABICT) has obvious advantages of open and transparent data, unchangeable and long-term operation. With its unique trust-building mechanism, it realizes penetrative supervision and step-by-step trust transfer, which has great potential for innovation [2]. The establishment of the equipment quality management based on ABICT means that the department and the task team can establish a "seamless link" through intelligent and efficient and control of the equipment, thus realizing the visualization, intelligence and actual combat.

\section{ABICT COMPOSITION AND PRINCIPLE}

\subsection{Basic Structure of Alliance Blockchain}

Blockchain is a kind of accounting technology, which is mutually maintained by many parties, uses cryptography to ensure safe transmission and access, and can achieve consistent data storage, difficult tampering and non-repudiation prevention. It is also called distributed ledger technology [3]. Blockchain operates in one way through blocks and the time chain for generating transaction records [4]. According to its principle and mechanism, it can be divided into public chain, alliance chain and private chain from the 
perspective of the scale of distributed network and the control of data reading and writing authority [5]. The basic structure principle of alliance chain is shown in Figure 1.

\subsection{Working Principle of Alliance Chain}

Alliance chain is called "partially decentralized" blockchain. Although its writing permission is limited within a certain institution, it can realize decentralization among single institutions, thus ensuring the openness, transparency and non-tampering of data [6]. It has the characteristics of openness and low public trust, privacy protection and single high trust of private chain, so it is easy to set control authority, simpler and more efficient process and higher scalability.

\subsection{The Principle of ABICT}

ABICT is a computer protocol that disseminates, verifies or executes contracts by means of information technology. It allows credible transactions without a third party, but these transactions are traceable and irreversible, thus ensuring that the whole process is open, transparent and unchangeable as well as avoiding the influence of centralized institutions. The application of ABICT intelligent contract technology can avoid the participation of centralized servers, which means saving social resources, reducing transaction steps and time, and solving credit problems.

\subsection{Technical Advantages of ABICT Advantages}

The technical characteristics of ABICT are mainly manifested in the following aspects. First, the data is open and transparent, and any party can check its code and data at runtime. Second, data cannot be altered, and nodes running smart contracts need not worry about other nodes maliciously modifying code and data. Third, the data can run for a long time, and the failure of the nodes supporting the alliance chain will not lead to the stop of the intelligent contract, and its reliability theoretically operates permanently and is valid all the time. Because of its obvious advantages, as shown in Figure 2, according to the requirements of equipment tasks, computer protocols are automatically functioned to determine whether to carry it out or not.

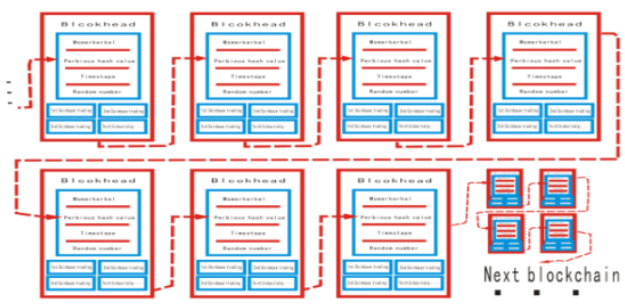

Figure 1 Basic structure diagram of alliance chain.
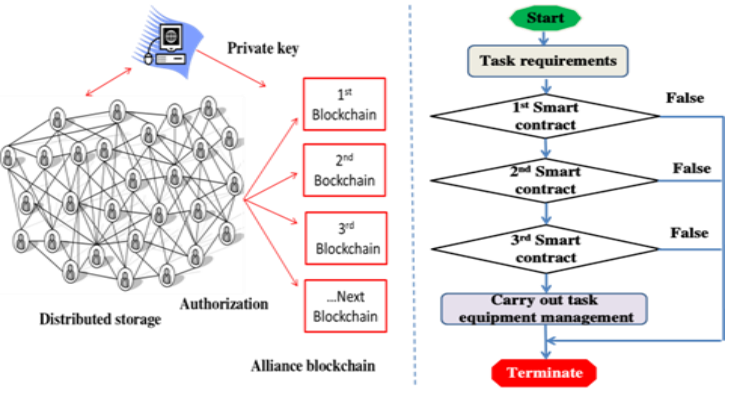

Figure 2 Working principle and process of ABICT.

\section{ANALYSIS OF EQUIPMENT MANAGEMENT FOR ACCOMPANYING TASKS BASED ON ABICT}

According to the definition of quality in ISO9000 quality management system, GJB9001B and GJB1405A equipment quality management terminology, Equipment quality [7] can be understood as the degree to which a set of inherent characteristics of equipment meet customer requirements. The quality management of equipment for carrying out tasks, that is a series of coordination activities organized by the command and control in carrying out tasks. The basic requirements of its mode based on ABICT, its basic requirements include three aspects, namely, improving the efficiency of equipment allocation, arrangement, delivery, maintenance and operation, improving the level of actual combat support of equipment and effectively reducing the cost of equipment quality information supervision. In order to meet the above-mentioned efficiency, it is necessary to construct the quality management model.

The first point is to construct the operation specification of alliance chain. During the mission, scientific research units, equipment management departments, equipment manufacturers and equipment users are integrated and brought up by the consensus mechanism of alliance chain POS, POW, POA and PBFT under the support of ABICT. All of its participate in the update and maintenance of combat and technical status of mission equipment, establish a new management model and organizational structure. At the same time, we should strengthen demand traction and matching, realize the interactive transformation of information within the organization, and maximize the efficiency of equipment quality management.

The second point is to collect feedback and suggestions in time. It can promote the further growth, progress and perfection of alliance chain. When the user puts forward the requirement of improving performance, the equipment manufacturer will improve the performance index according to the requirement in order to gain competitive advantage. At the same time, RFID anti-counterfeiting technology and ABICT are combined to trace the equipment quality data in the 
whole process, following integrity, high efficiency and informationization. By using PDCA cycle method, excellent performance management, Six Sigma management, QC group, Pareto chart, histogram and other methods, the equipment quality information can be effectively measured, controlled and improved, and the equipment quality management can be promoted.

The third and most important point is to refine the terms and conditions of smart contracts. Focus on certification, strengthen quality information management, and record and archive equipment information timely and accurately. During the team's implementation of major tasks, the poor environment and high utilization intensity of equipment inevitably affect the quality of equipment use. At this special moment, it is necessary to record all kinds of quality information of equipment in time and accurately, such as transportation, testing and use environment. By analyzing and evaluating various performance and quality states, ABICT rules are refined and loaded on the treaty rules, thus providing all-round support for commanders' decision-making.

\section{APPLICATION OF EQUIPMENT QUALITY MANAGEMENT BASED ON ABICT}

With the intensification and upgrading of global competition, the future development of blockchain technology becomes more and more fierce. Whoever can truly grasp the development trend of blockchain application can easily win in the future. In recent years, the western countries, led by the United States, have accelerated their research on the military application of blockchain. They try to use blockchain technology to create a safe and complete information guarantee system, which occupies the global first-Mover advantage [8]. Its application trend is shown in Figure 3.

At the same time, we are actively working towards this aspect, and applying ABICT to the quality management of tasks. It can store the quality information data in the blockchain on each node, and act through time stamps, thus realizing the scene application model as shown in Figure 4. That is, programmable operation of equipment management, encryption of data information, digitization of warehouse management, traceability of quality information and standardization of enterprise qualification certification in integration of defense and civilian technologies. This model improves the safety and integrity of the quality management of the completed tasks to a certain extent.
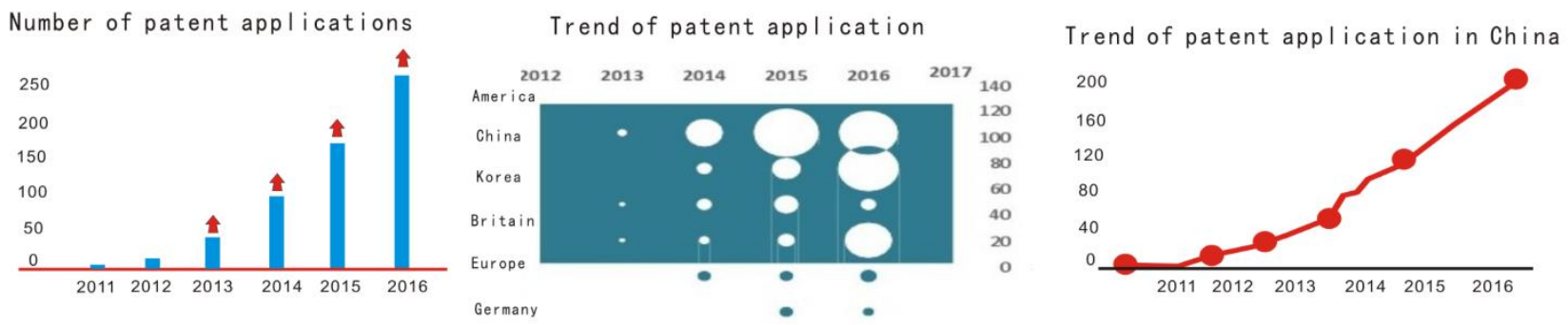

Figure 3 Development and application trend of blockchain.
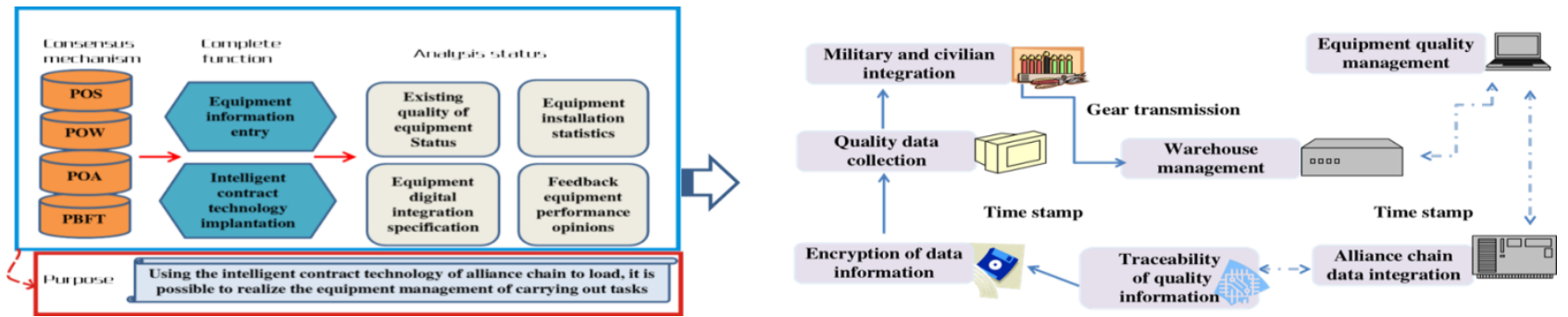

Figure 4 ABICT scenario application class model. 


\subsection{Establish Equipment Quality Management File Data Standard}

Establish quality information files by classification, grading and stratification, which can be queried at any time to provide data for adjusting and controlling the current actual quality. It mainly includes equipment quality report, quality information summary report, quality information feedback sheet, quality information feedback registration form, quality information measure completion report, visiting user report, user service report, comprehensive statistical report, feedback sheet for handling equipment quality user opinion case, user opinion request, market survey report, process quality table, operation instruction, equipment periodic inspection record card, equipment monthly spot inspection record card, tooling periodic inspection record card and measuring tool periodic verification card. Because the information data transfer in the blockchain will be accurately recorded by the time stamp in the system and can be permanently saved, the optimal economic effect of equipment management "people flow, logistics and information flow" [9] can be realized.

\subsection{Optimize the Distribution Control of Equipment Quality Management Structure}

By refining the rules and regulations and relevant provisions of ABICT, formulate detailed policies, regulations, standards and norms for quality management, as well as quality information management regulations and relevant working rules, etc. It can create a four-in-one three-dimensional supervision link of equipment setting, development, distribution, use, maintenance and training for manufacturers, competent departments, management departments and users. In that way, equipment manufacturers, higher authorities, equipment management departments and equipment users are encouraged to effectively participate in the update, maintenance and management of the equipment for the mission. Establishing a distributed and supervised three-dimensional equipment management structure is convenient for personnel verification and identity verification. All parties work under the direct leadership of the corresponding decision-making bodies.

\subsection{Sharing Equipment Management Optimized Allocation of Resources in Integration of Defense and Civilian Technologies}

The characteristics of alliance chain, such as public point-to-point transmission, distributed storage, traceability and non-tampering, are conducive to efficient information sharing and resource raising, and optimize access control authority. By calling ABICT and storing it in the chain, each node in the chain interweaves with each other, and all the nodes jointly maintain the equipment quality data information generated in the sharing [10], and establish the chain group of equipment quality management alliance for carrying out tasks as shown in Figure 5. It can effectively solve the malicious operation behavior, increase the credibility and security of data sharing, and realize the effective docking and rapid mobilization between military and local. So as to avoid the situation of inaccurate information storage and exchange flow or data leakage and loss. To a certain extent, it can improve the efficiency of data information protection, equipment resource utilization efficiency and equipment quality management level in integration of defense and civilian technologies.

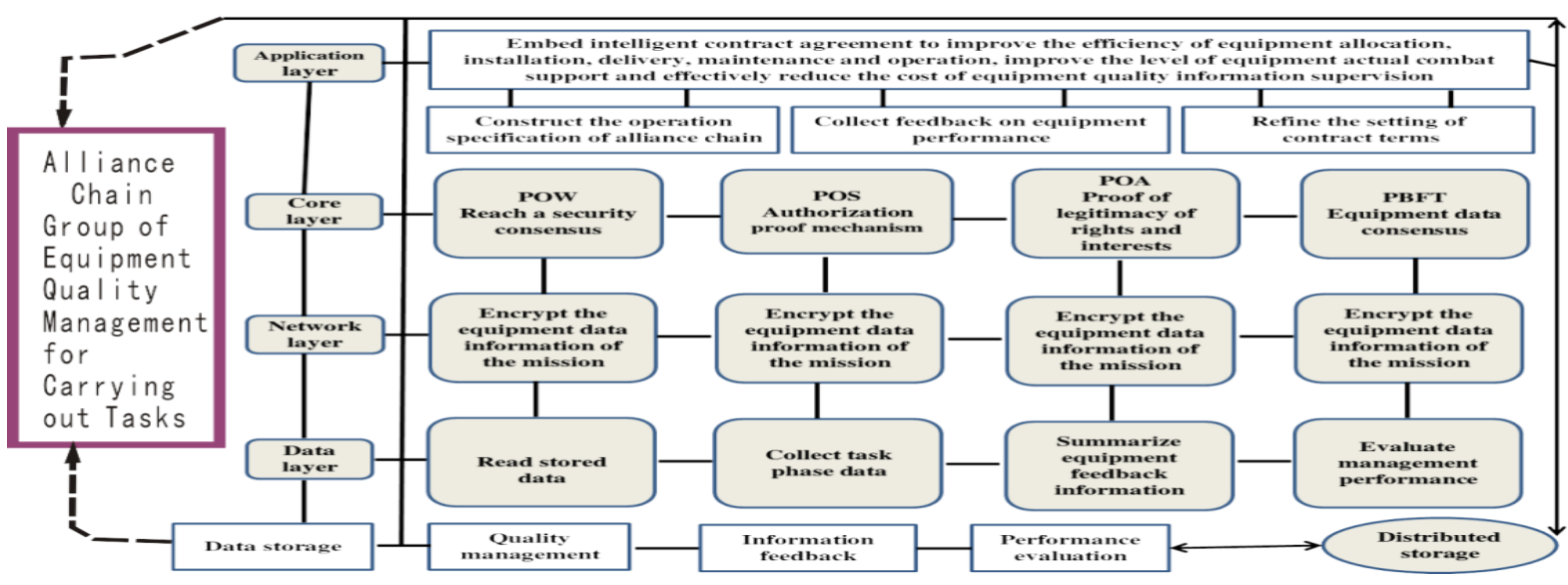

Figure 5 The overall framework of the group for carrying out the task. 


\section{CONCLUSION}

There are many technical and social challenges in the decision-making of equipment quality management development. Only by continuously improving and matching the equipment management application based on ABICT, can the "fusion effect" of guarantee delivery be produced, and the management mode of matching responsibility, right and accountability is established. It can build an effective common governance mechanism and promote all parties to participate in the decision-making process of equipment management development. This model can fully mobilize the subjective initiative of all parties. In practice, the optimal combination scheme of equipment architecture system for carrying out tasks is gradually formed. So as to ensure that the tasks of "housing, transportation, delivery and repair" can be quickly put in place, followed up in the whole process and continuously supplied.

\section{ACKNOWLEDGMENTS}

This work is supported by the Natural Science Basic Research Plan in Shanxi Province of China (No.2020JM-361), the Young and middle-aged scientific research backbone projects of Engineering University of PAP (No.KYGG201905) and the basic research foundation project of Engineering University of PAP (No.WJY201920, No.WJY202019), military theory research project of Engineering University of PAP (No.JLY2020085), education and teaching fund project of Engineering University of PAP (No.WJJ202039), the PAP's Military Scientific Research Mandatory Project (No.WJ2020A020047, No.WJ2020A020048, No.WJ2020A020029, No.WJ2020A020049).

\section{REFERENCES}

[1] Liu Xiaofang, Xie Yi, Equipment Life Cycle Quality Management. Beijing: National Defense Industry Press, June 2014.
[2] China Information and Communication Research Institute, Trusted Blockchain Promotion Plan, Blockchain White Paper (2019) . Beijing: China Information and Communication Research Institute and Trusted Blockchain Promotion Plan Press, October 2019.

[3] Yuan Yong, Wang Feiyue, Development status and prospect of blockchain technology. acted automatic silica, 2016, 42(4):481-494.

[4] Wu Xuchuan, Wang Peng, application and prospect of blockchain technology. Tsinghua financial review, 2016, (10):23-25.

[5] Fang Xingu, Implementation of Key Technologies of Trusted Degree Query System Based on blockchain. Dalian Maritime University, 2017.

[6] Wang Yangzi, Hour Joe, Zhang Yue, Data management based on blockchain technology. Electronic Design Engineering, 2019, (6):87-95.

[7] ANTONOPOULOSAM. Mastering Bitcoin: Unlocking Digital Crypto-currencies. California: O'ReillyMediaInc, 2015.

[8] Zhao Hue, Yan Ming, Unswervingly take the road of strengthening the army through science and technology with Chinese characteristics-study and implement President Xi's important exposition on the strategy of strengthening the army through science and technology. Research on the Theory of Military Political Work, 2017, 18(1):7-10.

[9] Zhao Mingus, Zhang Lu, Qi Jin, Trusted Service Management Framework of Social Internet of Things Based on blockchain. Telecommunications Science, 2017,(10):19-25.

[10] Wang Xiaohui, Chen Jun, Wang Yunfei. Research on Life Cycle Management of Oil Equipment Based on blockchain. Equipment Environmental Engineering, 2019, (11):100-105. 